\title{
Isolation of Fibroblast-Activation Protein-Specific Cancer-Associated Fibroblasts
}

\author{
Yingying Huang, ${ }^{1}$ Sufang Zhou, ${ }^{1}$ Yong Huang, ${ }^{1}$ Duo Zheng, ${ }^{2}$ Qiqi Mao, ${ }^{1}$ Jian He, \\ Yiwei Wang, ${ }^{1}$ Dabing Xue, ${ }^{1}$ Xiaoling Lu, ${ }^{1}$ Nuo Yang, ${ }^{1}$ and Yongxiang Zhao ${ }^{1}$ \\ ${ }^{1}$ National Center for International Research of Biological Targeting Diagnosis and Therapy, \\ Guangxi Key Laboratory of Biological Targeting Diagnosis and Therapy Research, Collaborative Innovation Center for \\ Targeting Tumor Diagnosis and Therapy, Guangxi Medical University, Nanning, Guangxi, China \\ ${ }^{2}$ Shenzhen Key Laboratory of Translational Medicine of Tumor, Department of Basic Medicine, School of Medicine, \\ Shenzhen, Guangdong 518000, China
}

Correspondence should be addressed to Yongxiang Zhao; yongxiang_zhao@126.com

Yingying Huang and Sufang Zhou contributed equally to this work.

Received 9 March 2017; Revised 9 May 2017; Accepted 15 May 2017; Published 14 August 2017

Academic Editor: Takashi Saku

Copyright (C) 2017 Yingying Huang et al. This is an open access article distributed under the Creative Commons Attribution License, which permits unrestricted use, distribution, and reproduction in any medium, provided the original work is properly cited.

\begin{abstract}
The current study is to develop a gentle and efficient method for purification of fibroblast-activation protein positive $\left(\mathrm{FAP}^{+}\right)$cancerassociated fibroblasts (CAFs) from tumor tissues. Fresh tissues were isolated from BALB/c-Nude mice bearing human liver cancer cell line (HepG2), fully minced and separated into three parts, and digested with trypsin digestion and then treated with collagenase type IV once, twice, or thrice, respectively. Finally, the cells were purified by using FAP magnetic beads. The isolated CAFs were grown in culture medium and detected for the surface expression of fibroblast-activation protein (FAP). The number of adherent cells which were obtained by digestion process with twice collagenase type IV digestion was $(5.99 \pm 0.18) \times 10^{4}$, much more than that with the only once collagenase type IV digestion $(2.58 \pm 0.41) \times 10^{4}(P<0.0001)$ and similar to thrice collagenase type IV digestion. The percentage of $\mathrm{FAP}^{+}$CAFs with twice collagenase type IV digestion (38.5\%) was higher than that with the only once collagenase type IV digestion (20.0\%) and little higher than thrice collagenase type IV digestion (37.5\%). The FAP expression of CAFs was quite different from normal fibroblasts (NFs). The fibroblasts isolated by the innovation are with high purity and being in wonderful condition and display the features of CAFs.
\end{abstract}

\section{Introduction}

Cancer-associated fibroblasts (CAFs) play an essential role in promoting tumor occurrence and growth [1-4]. Tumor proliferation, angiogenesis, invasion, and metastasis are dependent on the cancer-associated fibroblasts secretion of various cytokines, chemokines, growth factors, and degradation of extracellular matrix (ECM) proteins for platform [5-8]. Based on previous studies involving tumor stromal fibroblasts or extracellular matrix, the cancer-associated fibroblasts (CAFs) and normal fibroblasts (NFs) differ in biological characteristics and gene expression profile $[9,10]$. When compared to normal fibroblasts, cancer-associated fibroblasts are spindle- and stellate-liked cells, with abundant cytoplasm and poor cell polarity, growth dense, and uncontrolled, highly and specifically expressed $\alpha$-smooth muscle actin ( $\alpha$-SMA) [11]. Cancer-associated fibroblasts are responsible for the generating of cytokines and growth factors which can affect tumor cells growth and metastasis and the production and reconstruction of most extracellular matrix in tumor stroma [12]. The study of the mechanism of the development of cancer-associated fibroblasts plays a major role for tumor diagnosis and therapy. Although cancer-associated fibroblasts are the major components of cancer stroma, they are densely arranged and surrounded by connective tissue and various other cell types, embedded in matrix components [13]. Therefore, it is beset with difficulties to isolate cancerassociated fibroblasts from tumor tissue. 


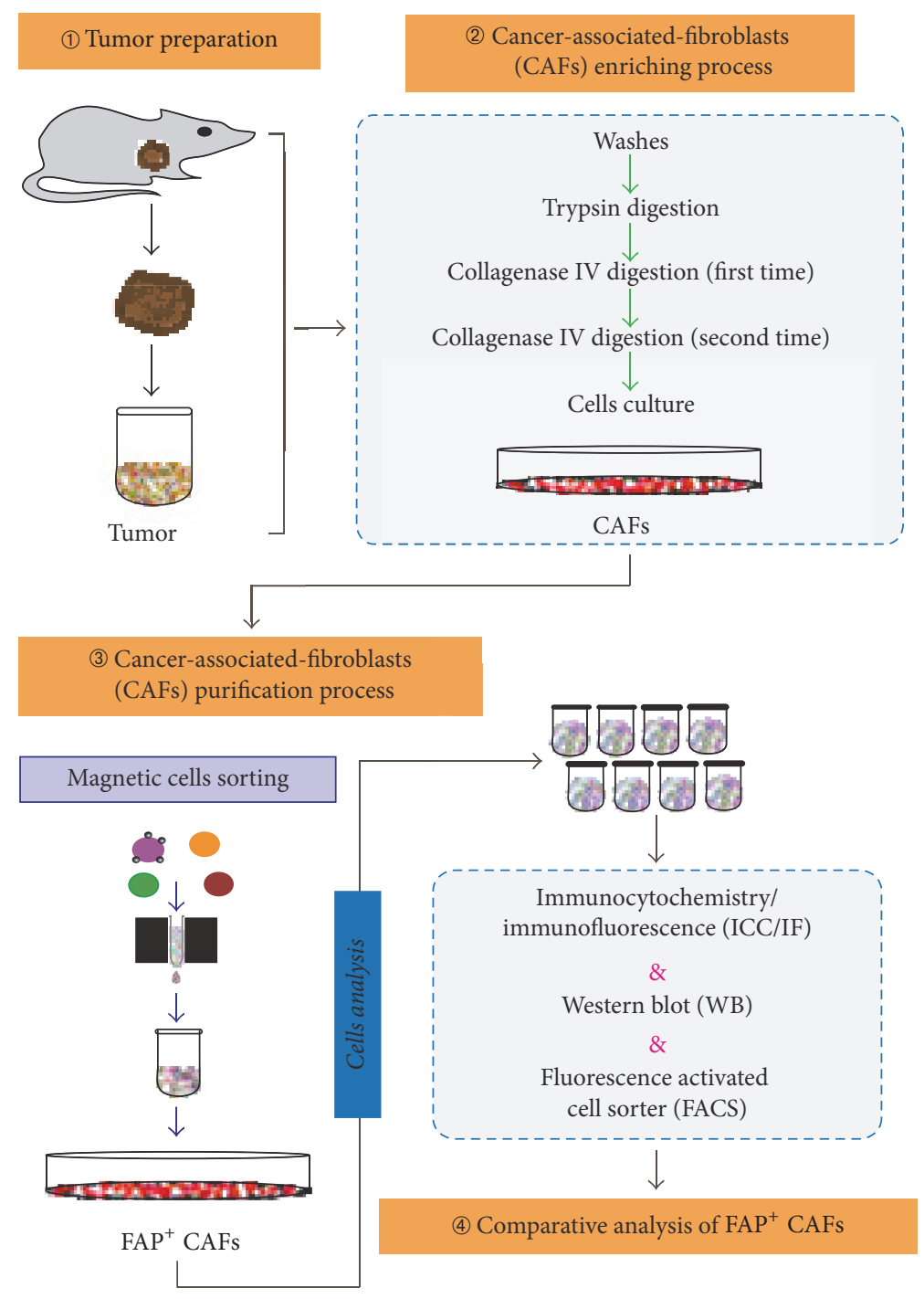

FIGURE 1: Flow chart and overview of the $\mathrm{FAP}^{+} \mathrm{CAFs}$ isolation procedure.

For the past few years, purification technology of CAFs for culture and molecular profiling has obtained more and more attention and become more and more diversified, such as using Fluorescence-Activated Cell Sorting (FACS) to isolate CAFs and NFs from fresh tissues; however, FACS is too expensive to popularize and may lose some cells during the process [14-16]. Besides, some researchers found that not all CAFs at all times express specific marker fibroblast-activation protein (FAP) [17]. These isolated techniques all tend to got only a small number of wanted cells. This paper reports a new purification method to gain numerous $\mathrm{FAP}^{+} \mathrm{CAFs}$ in good conditions.

\section{Material and Methods}

2.1. Cells and Animals. BALB/c-Nude mice were purchased from Vital River Company (Beijing, China) and were housed and cared in accordance with the Federation of European Laboratory Animal Science Association guidelines, and all protocols were approved by the Animal Ethics Committee of Guangxi Medical University (Nanning, Guangxi, China). Human liver carcinoma cells (HepG2) $\left(1 \times 10^{6}\right)$ were injected into the left flank of mice. 50 days after injection, tumors were excised for study.

2.2. Cancer-Associated Fibroblasts (CAFs) Isolation (Figure 1). Tumors were separated and placed in $37^{\circ} \mathrm{C}$ PBS solution for 1-3 min. Peripheral and necrotic tissues were removed and remaining tumor was fully cut up by using an ophthalmic scissor. Dissociation of $0.2 \times 0.2 \times 0.2 \mathrm{~cm}^{3}$ minced tissues was digested in $5 \mathrm{ml}$ trypsin solution $\left(\mathrm{DDH}_{2} \mathrm{O}, 40 \mathrm{mg} \mathrm{NaCl}\right.$, $2.5 \mathrm{mg} \mathrm{NaHCO}, 5 \mathrm{mg}$ glucose, $1.5 \mathrm{mg}$ ethylenediaminetetraacetic acid (EDTA), and $1.25 \mathrm{mg}$ trypsin) at room temperature for $5 \mathrm{~min}$ and then ended by $10 \mathrm{ml} \mathrm{FBS}$. These suspended tissues were washed by PBS solution and then were resuspended in an enzyme cocktail of $20 \mathrm{ml}$ Dulbecco's modified Eagle's medium (DMEM), $2 \mathrm{ml} \mathrm{FBS}$, and $10 \mathrm{mg}$ collagenase 
type IV at room temperature for $5,10,20,30,45,60$, and 75 min of constant mixing with a vortex. Tissues were gently pipetted by using a pasteur pipette, PBS solution washed, and then were resuspended in $10 \mathrm{~mL}$ DMEM medium (containing $10 \% \mathrm{FBS}$ ), plated into $25 \mathrm{~cm}^{2}$ cell culture flasks and cultured in incubator $\left(37^{\circ} \mathrm{C}, 5 \% \mathrm{CO}_{2}\right)$ for two days. During this time, through the microscope, some adherent cells were found climbing out from these suspension loose tumor tissues. The rest of the suspension loose tumor tissues was through the above digestion process for the second time and the third time. Single cells were magnetically labeled with anti-FAP microbeads (Miltenyi Biotec, Bergisch Gladbach, Germany) in the dark at $4^{\circ} \mathrm{C}$ for $30 \mathrm{~min}$ and applied to the prepared MS Column (Miltenyi Biotec, Bergisch Gladbach). FAP ${ }^{-}$cells were collected in the flow-through of the column, while $\mathrm{FAP}^{+}$ cells bound to the beads were flushed out by applying the plunger supplied with the column. Sorted $\mathrm{FAP}^{+}$cells were plated into six-well plates and cultured in DMEM medium (containing 10\% FBS, ScienCell, USA).

2.3. Immunocytochemistry/Immunofluorescence (ICC/IF). Adherent cells that have been isolated were plated into sixwell plates at $1 \times 10^{5}$ cells/dish. At $70 \%$ confluence, serumfree DMEM was used as culture medium for $24 \mathrm{~h}$. Cells were washed and fixed with $4 \%$ paraformaldehyde and then incubated in $10 \%$ normal serum from the secondary antibody species at room temperature for 30 min to block nonspecific protein-protein interactions. Cells were then incubated with primary antibody FAP or $\alpha$-SMA $(1 \mu \mathrm{g} / \mathrm{ml})$ (Abcam, Cambridge, UK) at $4^{\circ} \mathrm{C}$ overnight. Then this was followed by Alexa Fluor 594-conjugated secondary (1/400) or Alexa Fluor 488-conjugated secondary (1/400) (Abcam, Cambridge, UK) at room temperature for $30 \mathrm{~min}$ and DAPI staining for $3 \mathrm{~min}$. The antigen-antibody binding was assessed with fluorescence microscopy.

2.4. Fluorescence-Activated Cell Sorting (FACS). For flow cytometry, cells were stained at the concentration of $1 \times 10^{6}$ cells $/ 90 \mu \mathrm{l}$ buffer and $10 \mu \mathrm{l}$ phycoerythrin-conjugated antibody FAP (R\&D Systems, Minneapolis, US) at $4^{\circ} \mathrm{C}$ for $30 \mathrm{~min}$ before FACS analysis. All data were analyzed by EXPO32 Software.

2.5. Western Blotting. Cells were washed at $4^{\circ} \mathrm{C}$ and lysed in RIPA buffer on ice. Then, cell lysates were centrifuged at $13,000 \mathrm{~g}$ at $4^{\circ} \mathrm{C}$ for $20 \mathrm{~min}$. Total protein concentration in the cell lysate was measured by BCA Protein Quantification kit (Beyotime Biotechnology). Cell lysates were separated by SDS-PAGE and transferred onto a nitrocellulose membrane. The nitrocellulose membrane was incubated with primary anti-FAP antibody at $4^{\circ} \mathrm{C}$ overnight. After washing the nitrocellulose membrane with TBS-T three times for $10 \mathrm{~min}$ each at room temperature, the nitrocellulose membrane was incubated with horseradish peroxidase- (HRP-) conjugated anti-IgG Abs (ZSGB-Bio, Beijing, China). Protein band intensities were quantified by use of the diaminobenzidine (DAB) kit (SolarBio, Beijing, China). $\beta$-Actin was used as internal control.
2.6. Statistical Analysis. Data are showed as mean \pm SEM. The significance of differences between groups was assessed by $t$-test. All analyses were performed with GraphPad Prism program version 5 (GraphPad Software, California, USA).

\section{Results}

3.1. Isolation of CAFs. Normal fibroblasts (NFs) isolated from normal human skin were small spindle cells, while CAFs colonies from tumor were stelliform cells with higher $\alpha$-SMA expression (Figure 2(a)). FAP $^{+}$cells reached highest level when collagenase type IV digested for $45 \mathrm{~min}$ with a vortex (Figure 2(b)). Before magnetic separation, the number of adherent cells digested with twice collagenase type IV digestion $\left((5.99 \pm 0.18) \times 10^{4}\right)$ was much higher than the one with only once collagenase type IV digestion $\left((2.58 \pm 0.41) \times 10^{4}\right.$, $P<0.0001)$ similar to the number of thrice collagenase type IV digestion $\left((6.09 \pm 0.50) \times 10^{4}\right.$, NS, not significant $)$ (Figure 2(c)).

3.2. Enrichment and Purity of $\mathrm{FAP}^{+} \mathrm{CAFs}$. Before purification by magnetic beads, the percentage of $\mathrm{FAP}^{+}$CAFs digested with twice collagenase type IV digestion (38.5\%) was much higher than that with only once collagenase type IV digestion $(20.0 \%)$ and little higher than the number of thrice collagenase type IV digestion (37.5\%) (Figure 3). After magnetic cell separation (MACS), we showed that $\mathrm{FAP}^{+}$CAFs are combined with microbeads as detected under a microscope (Figure 4).

3.3. Detection of $\mathrm{FAP}^{+}$CAFs Activity. Immunocytochemistry/immunofluorescence (ICC/IF) showed that FAP ${ }^{+}$CAFs after digestion with twice collagenase type IV digestion and purification by magnetic beads exhibited strong fluorescence in cytoplasm (Figure 5(a)). The purity of the FAP ${ }^{+}$CAFs was 99.68\%, while NFs which were isolated from normal human skin without FAP expression (Figures 5(b) and 5(c)).

\section{Discussion}

In the process of the tumor occurrence and growth, tumorassociated fibroblasts are emerged as essential inducement [18]. Moreover, the tumor microenvironment created by tumor-associated fibroblasts is always dominant during the process of tumor development. It is well known that, during tumor development, tumor-associated fibroblasts not only undergo cellular and molecular changes but also affect other normal stromal cells transformation, which accompany the appearance of tumor malignancy and invasion [19-21].

Cancer-associated fibroblasts, a major component of the cancer stroma, are necessary for tumor growth and metastasis $[22,23]$. FAP, $\alpha$-SMA, FSP1, and PDGFR are not only markers that have important role in cancer-associated fibroblasts but also essential for the proliferation of tumor cells and stimulating metastasis of tumors [17, 24].

In view of the known heterogeneity of tumor-associated fibroblasts, it would appear reasonable to study tumorassociated fibroblasts isolated from tumor tissues when studying the mechanisms of tumor progression [25-27]. 

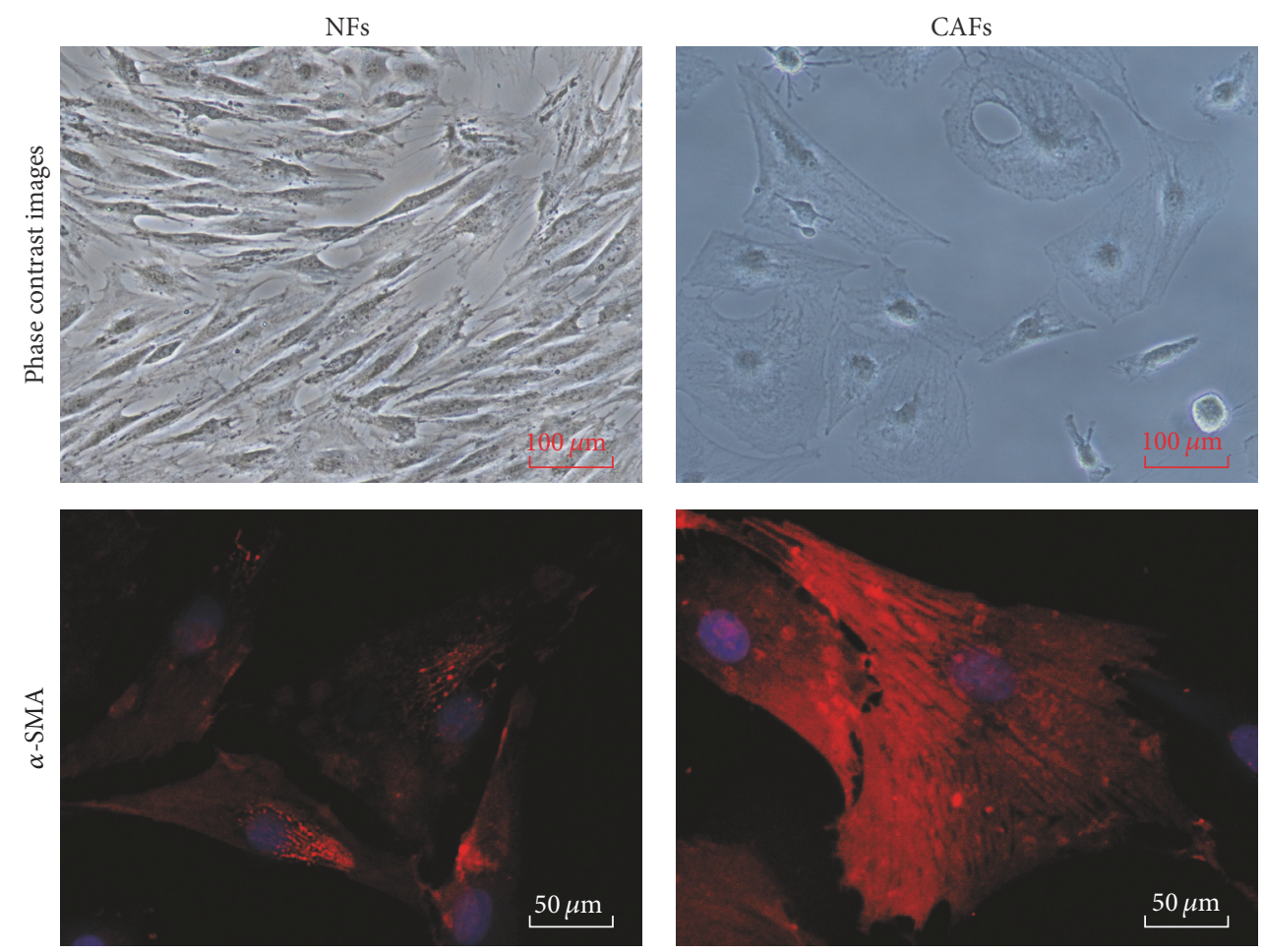

(a)

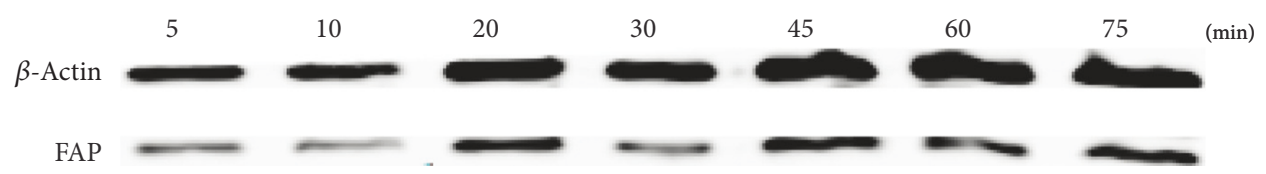

(b)

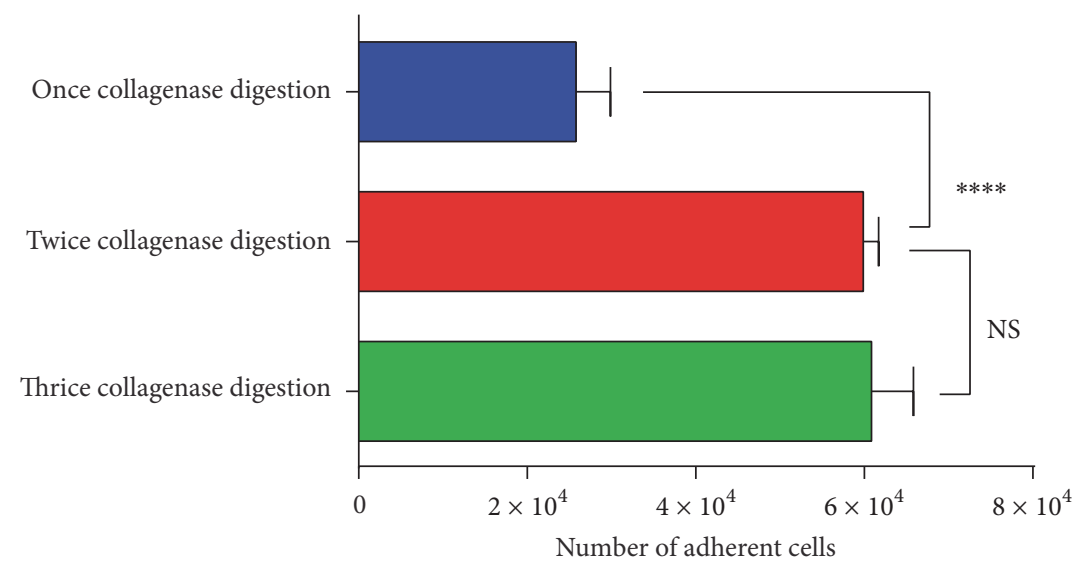

(c)

Figure 2: Cancer-associated fibroblasts (CAFs) colonies from tumor before purification by magnetic beads. (a) Phase contrast images and $\alpha$-SMA expression of CAFs after isolation by different digestion procedures. Normal fibroblasts (NFs) as negative control cells isolated from normal human tissue (such as skin) (scale bars represent $100 \mu \mathrm{m}$ and $50 \mu \mathrm{m}$ ). (b) Western blot analysis showed the FAP expression of adherent cells by different time duration of collagenase type IV digested with a vortex. (c) The statistical chart of the number of adherent cells after isolation by different digestion procedures. Data are presented as the mean $\pm \operatorname{SEM}(n=3)$. NS, not significant; ${ }^{* * * *} P<0.0001$.

Even though methods have been reported for the isolation of cancer-associated fibroblasts, the purity and efficiency of sorting have not been described.

In the present work, we reported a method to isolate $\mathrm{FAP}^{+}$ CAFs, an important cell population found in tumor tissue, through twice collagenase type IV digestion and magnetic cell separation. This purification technique produces isolated cells with purity in excess of $99.68 \%$, and much more number and better condition of $\mathrm{FAP}^{+}$CAFs than that with once and thrice collagenase type IV digestion. The specific markers 


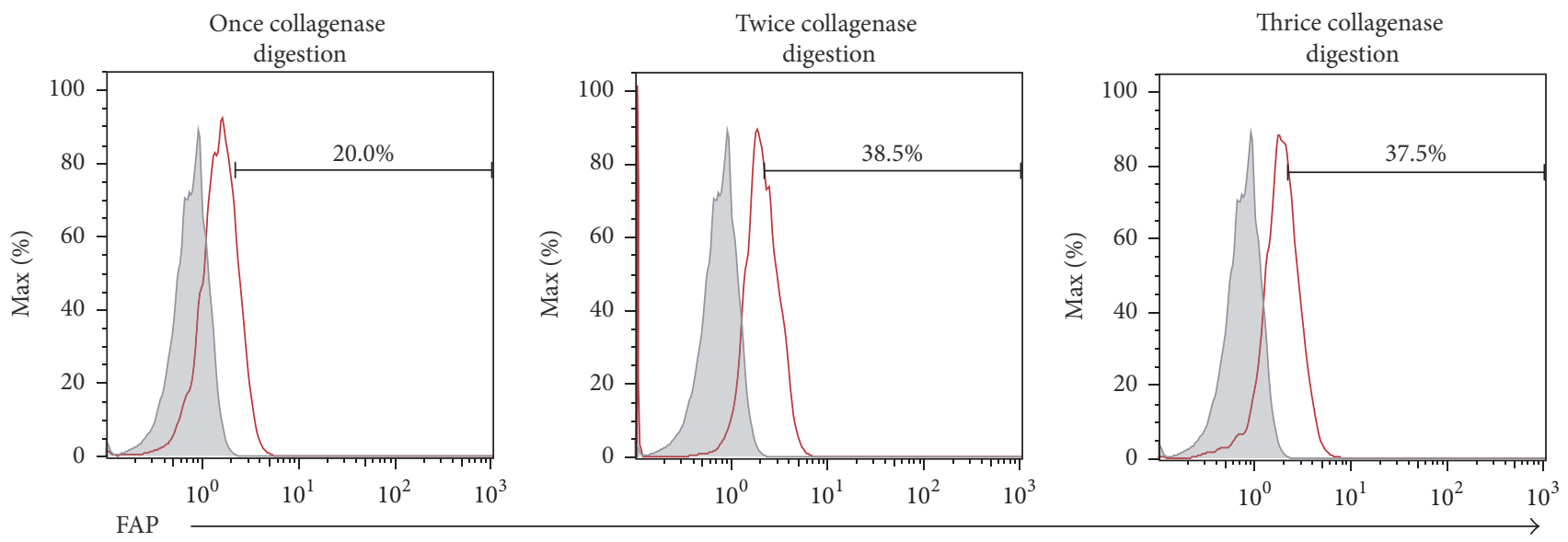

(a)
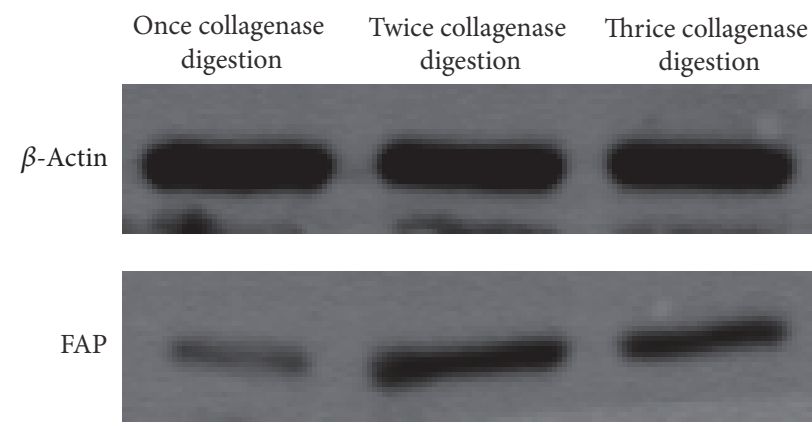

(b)

FIGURE 3: FAP expression of CAFs before purification by magnetic beads. (a) Fluorescence-Activated Cell Sorting (FACS) showed the FAP expression of CAFs. (b) Western blot analysis showed the FAP expression of CAFs.
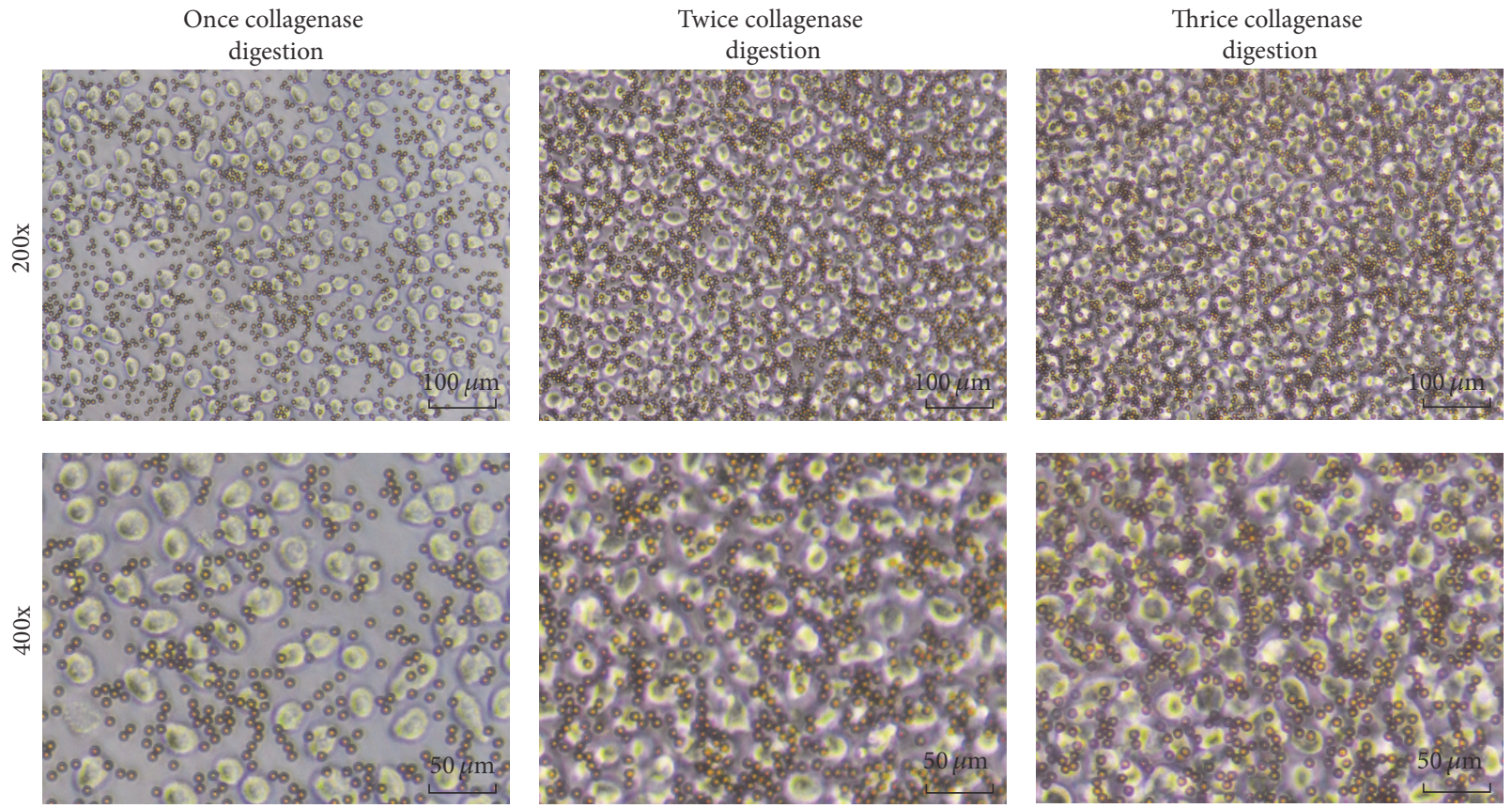

FIgURE 4: CAFs combined with magnetic beads (scale bars represent $100 \mu \mathrm{m}$ and $50 \mu \mathrm{m}$ ). 

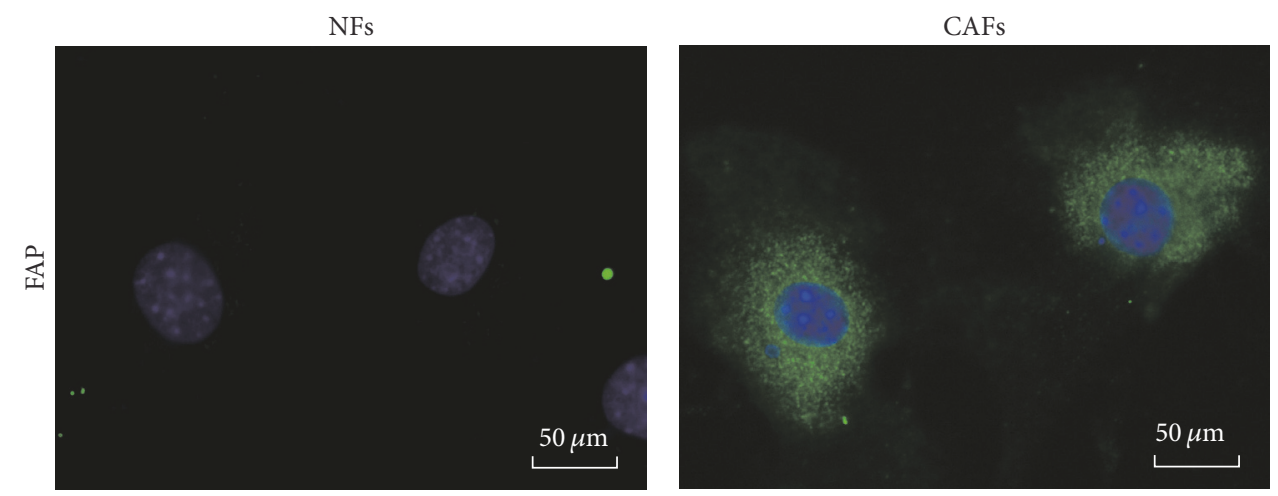

(a)
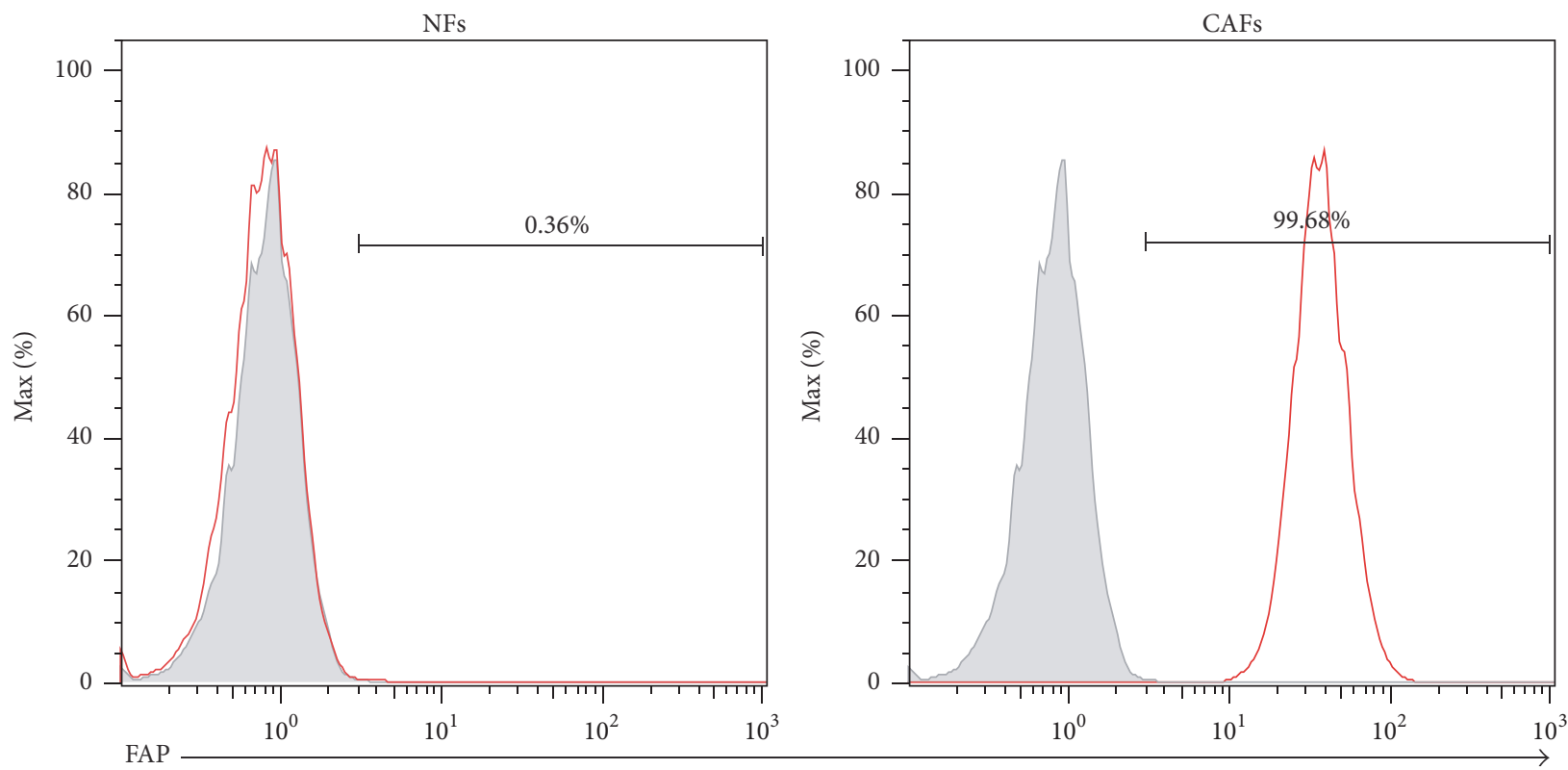

(b)

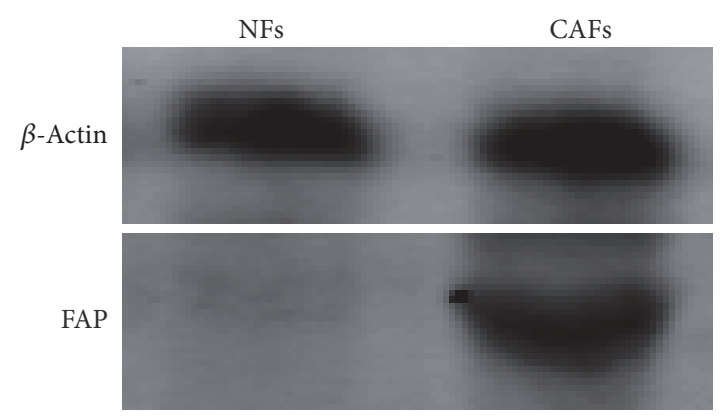

(c)

FIGURE 5: FAP expression of CAFs after purification by magnetic beads. (a) Immunocytochemistry/immunofluorescence (ICC/IF) results of FAP $^{+}$CAFs (scale bars represent $50 \mu \mathrm{m}$ ). (b) Fluorescence-Activated Cell Sorting (FACS) showed the FAP expression of CAFs. (c) Western blot analysis showed the FAP expression of CAFs. Normal fibroblasts (NFs) as negative control cells isolated from normal human tissue (such as skin). 
of cancer-associated fibroblasts (CAFs) are diametrically opposite to normal fibroblasts (NFs). This technology counts a great deal role in carcinogenesis.

\section{Conflicts of Interest}

The authors report no conflicts of interest in this work.

\section{Authors' Contributions}

Yingying Huang, Qiqi Mao, Xiaoling Lu, and Yongxiang Zhao designed the experiments. Sufang Zhou, Yong Huang, Duo Zheng, Jian He, Yiwei Wang, and Dabing Xue performed the experiments. Yingying Huang, Qiqi Mao, Jian He, and Nuo Yang analyzed the data. Yingying Huang, Qiqi Mao, Xiaoling Lu, and Yongxiang Zhao wrote the manuscript. All authors read and approved the final manuscript.

\section{Acknowledgments}

This study was supported, in part, by grants from the National Natural Scientific Foundation of China (nos. 81430055 and 81372452), Programs for Changjiang Scholars and Innovative Research Team in University (no. IRT_15R13), the International Cooperation Project of the Ministry of Science and Technology of China (no. 2015DFA31320), the Project for Innovative Research Team in Guangxi Natural Science Foundation (2015GXNSFFA139001), the Project of Science and Technology of Guangxi (nos. 14125008-2-12 and 15990052-10), and the Research and Development of Reproductive Medical Products of the Association of Southeast Asian Nations (ASEAN) of China (Guangxi) (no. 14251001).

\section{References}

[1] M. A. Frassanito, L. Rao, M. Moschetta et al., "Bone marrow fibroblasts parallel multiple myeloma progression in patients and mice: in vitro and in vivo studies," Leukemia, vol. 28, no. 4, pp. 904-916, 2014.

[2] G. Comito, E. Giannoni, C. P. Segura et al., "Cancer-associated fibroblasts and M2-polarized macrophages synergize during prostate carcinoma progression," Oncogene, vol. 33, no. 19, pp. 2423-2431, 2014.

[3] A. Tsuyada, A. Chow, J. Wu et al., "CCL2 mediates crosstalk between cancer cells and stromal fibroblasts that regulates breast cancer stem cells," Cancer Research, vol. 72, no. 11, pp. 2768-2779, 2012.

[4] L. Xiao, J. V. McCann, and A. C. Dudley, "Isolation and culture expansion of tumor-specific endothelial cells," Journal of Visualized Experiments, no. 104, p. e53072, 2015.

[5] T. Murata, H. Mizushima, I. Chinen et al., "HB-EGF and PDGF mediate reciprocal interactions of carcinoma cells with cancerassociated fibroblasts to support progression of uterine cervical cancers," Cancer Research, vol. 71, no. 21, pp. 6633-6642, 2011.

[6] S. Y. Ko, N. Barengo, A. Ladanyi et al., "HOXA9 promotes ovarian cancer growth by stimulating cancer-associated fibroblasts," Journal of Clinical Investigation, vol. 122, no. 10, pp. 3603-3617, 2012.

[7] C. D. Madsen, J. T. Pedersen, F. A. Venning et al., "Hypoxia and loss of PHD2 inactivate stromal fibroblasts to decrease tumour stiffness and metastasis," EMBO Reports, vol. 16, no. 10, pp. 1394-1408, 2015.

[8] Q. Mao, X. Huang, J. He et al., "A novel method for endothelial cell isolation," Oncology Reports, vol. 35, no. 3, pp. 1652-1656, 2016.

[9] G. Ishii, A. Ochiai, and S. Neri, "Phenotypic and functional heterogeneity of cancer-associated fibroblast within the tumor microenvironment," Advanced Drug Delivery Reviews, vol. 99, pp. 186-196, 2016.

[10] B. Orr, A. C. P. Riddick, G. D. Stewart et al., "Identification of stromally expressed molecules in the prostate by tag-profiling of cancer-associated fibroblasts, normal fibroblasts and fetal prostate," Oncogene, vol. 31, no. 9, pp. 1130-1142, 2012.

[11] T. Ji, Y. Zhao, Y. Ding et al., "Transformable peptide nanocarriers for expeditious drug release and effective cancer therapy via cancer-associated fibroblast activation," Angewandte Chemie (International Edition in English), vol. 55, no. 3, pp. 1050-1055, 2016.

[12] M. M. Al-Ansari, S. F. Hendrayani, A. I. Shehata, and A. Aboussekhra, "P16(INK4A) represses the paracrine tumorpromoting effects of breast stromal fibroblasts," Oncogene, vol. 32, no. 18, pp. 2356-2364, 2013.

[13] F. Bruzzese, C. Hägglöf, A. Leone et al., "Local and systemic protumorigenic effects of cancer-associatedfibroblast- derived GDF15," Cancer Research, vol. 74, no. 13, pp. 3408-3417, 2014.

[14] V. Catalano, A. Turdo, S. Di Franco, F. Dieli, M. Todaro, and G. Stassi, "Tumor and its microenvironment: a synergistic interplay," Seminars in Cancer Biology, vol. 23, no. 6, pp. 522532, 2013.

[15] L. J. A. C. Hawinkels, M. Paauwe, H. W. Verspaget et al., "Interaction with colon cancer cells hyperactivates TGF- $\beta$ signaling in cancer-associated fibroblasts," Oncogene, vol. 33, no. 1, pp. 97-107, 2014.

[16] F. Sotgia, U. E. Martinez-Outschoorn, A. Howell, R. G. Pestell, S. Pavlides, and M. P. Lisanti, "Caveolin-1 and cancer metabolism in the tumor microenvironment: markers, models, and mechanisms," Annual Review of Pathology: Mechanisms of Disease, vol. 7, pp. 423-467, 2012.

[17] K. Shiga, M. Hara, T. Nagasaki, T. Sato, H. Takahashi, and H. Takeyama, "Cancer-associated fibroblasts: their characteristics and their roles in tumor growth," Cancers, vol. 7, no. 4, pp. 24432458, 2015.

[18] A. Calon, E. Espinet, S. Palomo-Ponce et al., "Dependency of colorectal cancer on a TGF- $\beta$-driven program in stromal cells for metastasis initiation," Cancer Cell, vol. 22, no. 5, pp. 571-584, 2012.

[19] E. Alspach, K. C. Flanagan, X. Luo et al., "P38MAPK plays a crucial role in stromal-mediated tumorigenesis," Cancer Discovery, vol. 4, no. 6, pp. 716-729, 2014.

[20] A. Hoshino, G. Ishii, T. Ito et al., "Podoplanin-positive fibroblasts enhance lung adenocarcinoma tumor formation: Podoplanin in fibroblast functions for tumor progression," Cancer Research, vol. 71, no. 14, pp. 4769-4779, 2011.

[21] A. E. Sirica, "The role of cancer-associated myofibroblasts in intrahepatic cholangiocarcinoma," Nature Reviews Gastroenterology and Hepatology, vol. 9, no. 1, pp. 44-54, 2012.

[22] T. Alkasaliasa, E. Flaberg, V. Kashuba et al., "Inhibition of tumor cell proliferation and motility by fibroblasts is both contact and soluble factor dependent," in Proceedings of the National Academy of Sciences of the United States of America, vol. 111, pp. 17188-17193, 2014. 
[23] J. Harper and R. C. A. Sainson, "Regulation of the anti-tumour immune response by cancer-associated fibroblasts," Seminars in Cancer Biology, vol. 25, pp. 69-77, 2014.

[24] S. Madar, I. Goldstein, and V. Rotter, "Cancer associated fibroblasts'-more than meets the eye," Trends in Molecular Medicine, vol. 19, no. 8, pp. 447-453, 2013.

[25] J. Albrengues, T. Bertero, E. Grasset et al., "Epigenetic switch drives the conversion of fibroblasts into proinvasive cancerassociated fibroblasts," Nature Communications, vol. 6, p. 10204, 2015.

[26] J. Paggetti, F. Haderk, M. Seiffert et al., "Exosomes released by chronic lymphocytic leukemia cells induce the transition of stromal cells into cancer-associated fibroblasts," Blood, vol. 126, no. 9, pp. 1106-1117, 2015.

[27] C. E. Weber, A. N. Kothari, P. Y. Wai et al., "Osteopontin mediates an MZF1-TGF- $\beta 1$-dependent transformation of mesenchymal stem cells into cancer-associated fibroblasts in breast cancer," Oncogene, vol. 34, no. 37, pp. 4821-4833, 2015. 


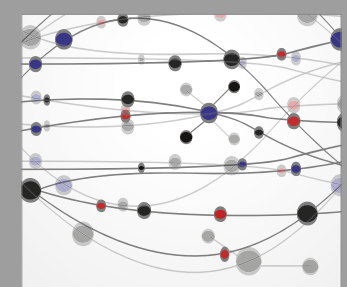

The Scientific World Journal
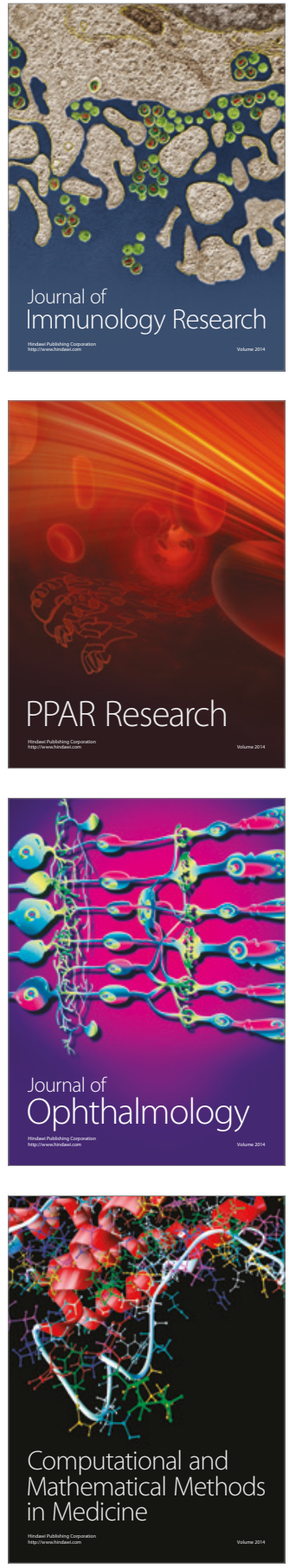

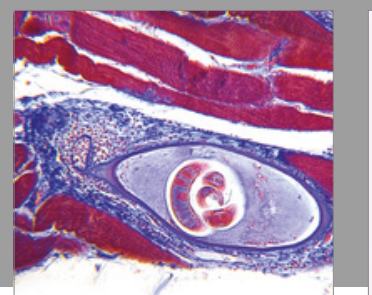

Gastroenterology Research and Practice
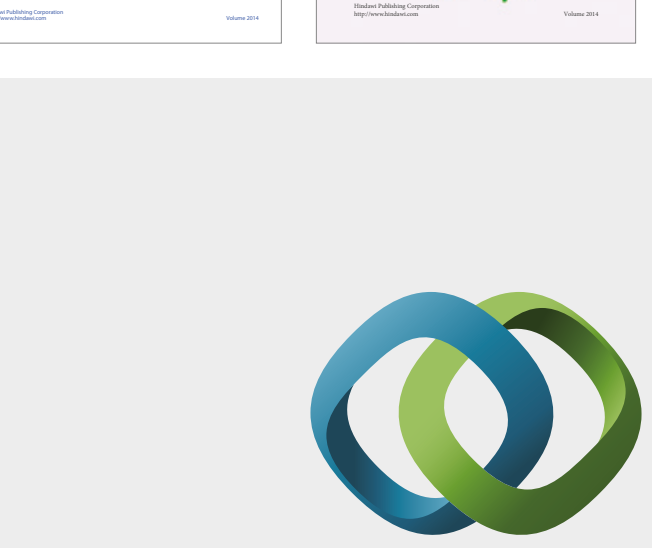

\section{Hindawi}

Submit your manuscripts at

https://www.hindawi.com
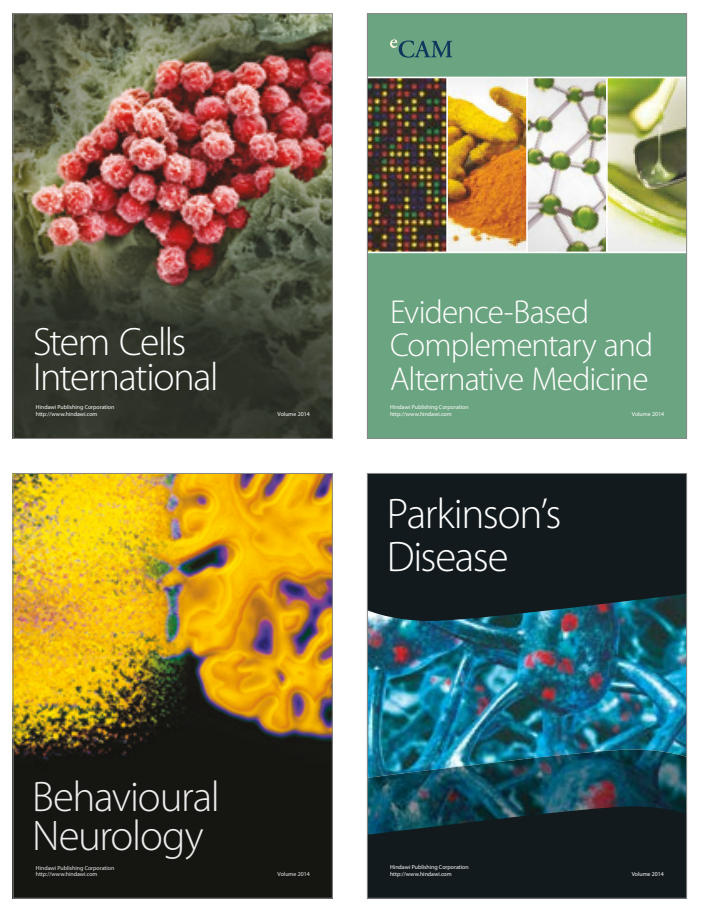
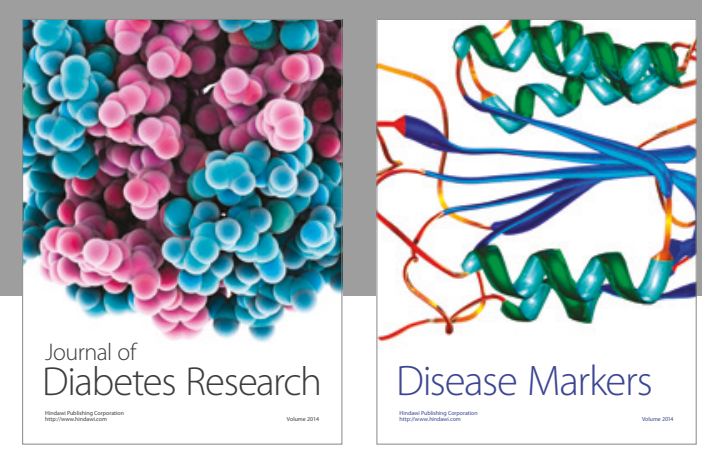

Disease Markers
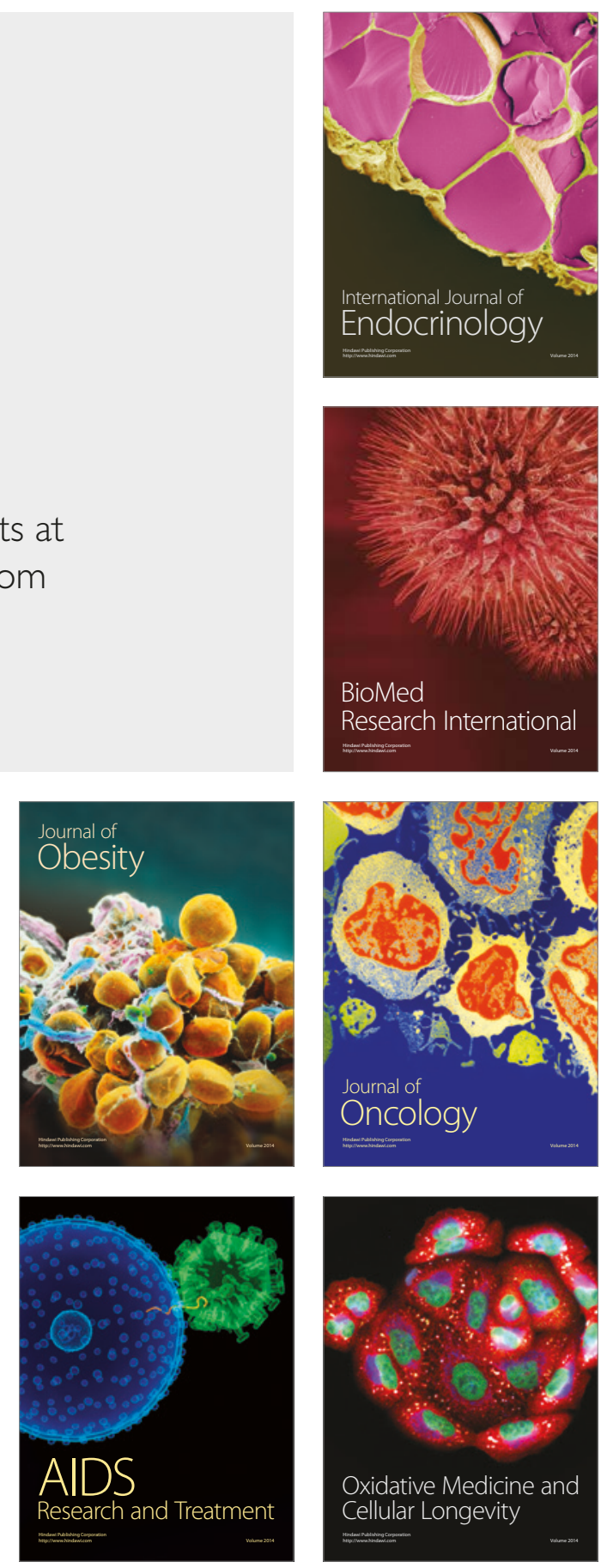